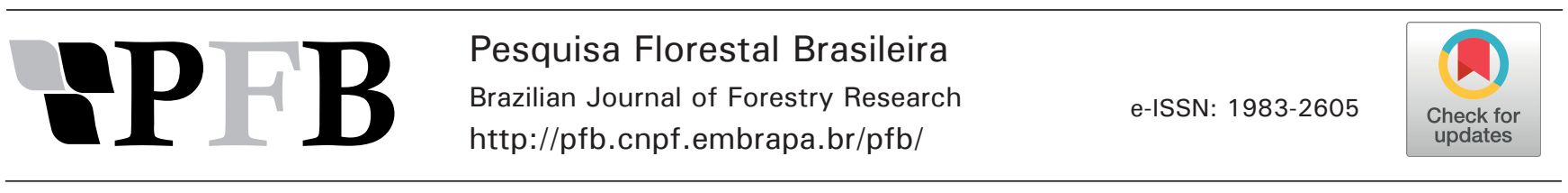

\title{
Fatores edafoclimáticos relacionados à produção de sementes em castanhais nativos de Roraima
}

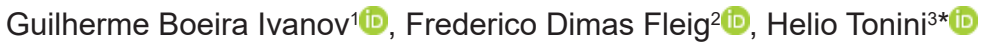 \\ ${ }^{1}$ Universidade Estadual do Maranhão, Rua Godofredo Viana, 1300, CEP 65900-100 Centro, Imperatriz, MA, Brasil \\ ¿Universidade Federal de Santa Maria, Avenida Roraima 1000, CEP 97105-900, Camobi, Santa Maria, RS, Brasil \\ ${ }^{3}$ Embrapa Pecuária Sul, Rodovia dos Pioneiros MT-222, Km 2,5, Zona Rural, CP. 343, CEP 78550-970, Sinop, MT, Brasil
}

*Autor correspondente:

helio.tonini@embrapa.br

Termos para indexação:

Produto florestal não madeireiro

Amazônia

Floresta tropical

Index terms:

Nom timber forest products

Amazon

Tropical forest

Histórico do artigo:

Received in 13/12/2017

Accepted in 10/10/2018

Published in 28/12/2018

doi: 10.4336/2018.pfb.38e201701553

\begin{abstract}
Resumo - Apesar da importância socioeconômica para a Amazônia, o conhecimento sobre os fatores que influenciam a produção de frutos e sementes da castanheira-dobrasil ainda é incipiente. Neste trabalho, avaliamos a influência da precipitação e textura, alagamento e profundidade do solo na produção de sementes. Os dados foram coletados em árvores nativas monitoradas em parcelas permanentes de 9 ha (300 m x $300 \mathrm{~m})$ no município de Caracaraí, RR, por cinco anos. Utilizamos a correlação de Pearson para selecionar o período de precipitação e concluímos que a precipitação do mês de setembro mostrou-se positivamente correlacionada com a produção de frutos. As variáveis de solos foram avaliadas por ANOVA. Observamos que maiores precipitações no mês de setembro, mês de transição entre a estação seca e chuvosa, se correlacionaram de forma significativa e positiva com a produção de sementes. Árvores em solos profundos e com textura franco-argilosa mostraram maior produção de sementes do que aquelas em solos pouco profundos.
\end{abstract}

\section{Soil-climatic factors related to seed production of Brazil nut trees in Roraima State, Brazil}

\begin{abstract}
Despite the socioeconomic importance for the Amazon, knowledge about the factors that influence the production of Brazil nuts is still incipient. In this paper we evaluate the influence of precipitation and soil texture, flood and depth on seed production. The data were collected in native trees monitored in permanent plots of 9 ha $(300 \mathrm{~m} \times 300 \mathrm{~m})$ in the municipality of Caracaraí, Roraima State, for five years. We used Pearson correlation to select the precipitation period and we conclude that the precipitation of September was positively correlated with fruit production. The soil variables were evaluated by ANOVA and we observed that higher precipitations in September, month of transition from dry to rainy season, correlated significantly and positively with seed production. Trees in deep soils with clay loam texture showed higher seed production than those in shallow soils.
\end{abstract}

\section{Introdução}

A castanha-do-brasil (Bertholletia excelsa Bompl.) é uma árvore da família Lecythidaceae, semidecídua, heliófila, característica da mata alta de terra firme, ocorrendo em determinados locais com grande densidade (Lorenzi, 2000). Seus indivíduos adultos são geralmente emergentes e suas copas estão acima do dossel da floresta (Zuidema, 2003).

As amêndoas produzidas por esta árvore são consideradas um dos principais produtos extrativistas oriundo das florestas nativas da Amazônia, que têm grande importância para a economia de diversas 
localidades e populações na Bolívia, Peru e na Amazônia Brasileira (Collinson et al., 2000; Ortiz 2002).

No Brasil, a produção se concentra em florestas nativas amazônicas localizadas na Região Norte. Apenas $2 \%$ da produção é oriunda de plantações (Homma et al., 2014), com cerca de dois milhões de pessoas tendo no extrativismo a sua principal atividade econômica (Instituto de Manejo e Certificação Florestal e Agrícola, 2016). Em 2016, a castanha-do-brasil foi o quarto produto florestal extrativista não madeireiro em importância econômica no Brasil, com produção de 34.644 mil t e receita de $\mathrm{R} \$ 110,1$ milhões. O estado do Amazonas foi o maior produtor, com $40 \%$ da produção total (IBGE, 2016).

Kainer et al. (2007) destacaram a importância de se conhecer as variações de produção e suas causas, ao afirmarem que para árvores de alto valor econômico, como B. excelsa, um nível máximo de frutificação e uma mínima variação interanual podem ser fatores decisivos no sucesso comercial. Porém, ainda pouco se conhece sobre a influência dos fatores edafoclimáticos sobre a produção de frutos da espécie.

Müller \& Caizavara (1989) e Zuidema (2003) afirmaram que a floração da castanheira-do-brasil está intimamente relacionada com as condições climáticas da região e ocorre no inicio da estação chuvosa. As árvores na região oeste da Amazônia (Acre) florescem primeiro do que no leste (Pará), provavelmente, devido à distribuição e ao período das chuvas (Maués et al., 2002).

Porém, Tonini (2011), ao estudar a fenologia da espécie em Roraima, concluiu que a floração e a frutificação são anuais, longas e sincrônicas e que, diferente das outras regiões na Amazônia, a floração ocorreu durante a época de menor precipitação, e a frutificação predominantemente no período chuvoso. Quanto ao período de desenvolvimento dos frutos até a dispersão (queda), autores como Müller \& Caizavara (1989), Zuidema (2003) e Tonini (2011) observaram variações entre 10 e 14 meses.

As condições de solo podem ter efeito ainda não quantificado sobre a frutificação da castanheira-do-brasil (Zuidema, 2003). As populações nativas da espécie se localizam, preferencialmente, em solos argilosos ou argilo-arenosos, com maior frequência nos de textura média até pesada. Müller \& Caizavara (1989) e Kainer et al. (2007) observaram que a capacidade de troca catiónica (CTC) e o P foliar apresentaram correlações positivas com o número de frutos produzidos por árvore.
Costa et al. (2017) observaram que as árvores com maior produção de frutos estavam associadas ao maior teor de Al trocável na camada de $0-20 \mathrm{~cm}$ do solo, bem como com menor $\mathrm{pH}$ e saturação por bases. Estas variáveis não foram consideradas como responsáveis por interferir no potencial produtivo da castanheira-dobrasil, mas serviram como indicativo de que as árvores mais produtivas contribuem com a remoção de bases trocáveis, favorecendo a acidificação do solo.

Neste trabalho, avaliamos a influência da precipitação e textura, alagamento e profundidade do solo na produção de sementes em castanhais nativos de Roraima.

\section{Material e métodos}

A área do estudo se localiza na cidade de Caracaraí, RR. Foram utilizadas quatro parcelas permanentes de 9 ha (300 m x 300 m), instaladas pela Embrapa Roraima com o objetivo de estudar a dinâmica na produção de frutos e sementes em populações nativas de castanheirado-brasil. Foram instaladas duas parcelas na Fazenda Pau Rainha, na região do projeto de assentamento do Itã $\left(01^{\circ} 48^{\prime} 58,0^{\prime \prime} \mathrm{N}\right.$ e $\left.61^{\circ} 07^{\prime} 41,0^{\prime \prime} \mathrm{W}\right)$. As demais foram instaladas em um plano de manejo florestal na Fazenda Mundo Novo, na região do projeto de assentamento do

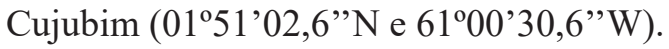

O clima da região é classificado como Ami (tropical chuvoso com pequeno período de seca), com precipitação média anual entre $1.700 \mathrm{~mm}$ a $2.000 \mathrm{~mm}$ e temperatura média anual de $27^{\circ} \mathrm{C}$. As chuvas são mais frequentes entre abril e agosto, sendo setembro caracterizado como mês de transição por apresentar redução marcante na precipitação. O período caracteristicamente seco ocorre entre novembro a março (Fundação do Meio Ambiente Ciência e Tecnologia do Estado de Roraima, 1993). Os solos predominantes são os argissolos amarelo e argissolos vermelho-amarelo, com afloramentos rochosos na área da Fazenda Mundo Novo.

Nas parcelas permanentes, todas as árvores com diâmetro a $1,30 \mathrm{~m}$ do solo (DAP) $>10 \mathrm{~cm}$ foram identificadas, mapeadas e numeradas com placas de alumínio. Após, foram selecionadas árvores consideradas produtivas, com DAP $>50 \mathrm{~cm}$, dominantes, com boa forma de copa e baixa infestação por cipós (Tabela 1).

A produção de frutos e sementes foi quantificada pela contagem do número de frutos e pelo peso fresco de sementes $\left(\mathrm{kg}_{\text {arvore }}{ }^{-1}\right)$. Todos os frutos em cada árvore amostrada foram contados e pesados em duas ocasiões 
durante a estação de queda dos frutos, que em Roraima ocorre entre março e julho. A abertura de todos os frutos e a pesagem das sementes foi realizada em campo, com a utilização de uma balança digital com precisão de 50 g. O período de monitoramento foi de cinco anos (entre 2006 a 2010), na fazenda Pau Rainha e de dois anos na a Fazenda Mundo Novo (2009 e 2010).

Tabela 1. Dados dendrométricos para os diferentes sítios e parcelas permanentes e o percentual de árvores de Bertholletia excelsa amostradas em Caracaraí, RR.

Table 1. Dendrometric data for the different sites and permanent plots and percentage of Bertholletia excelsa trees sampled in Caracaraí, Roraima State.

\begin{tabular}{cccccc}
\hline Local & Parcela & $\begin{array}{c}\text { DAP } \\
\text { (cm) }\end{array}$ & $\begin{array}{c}\mathbf{N} \\
\text { (árvores) }\end{array}$ & $\begin{array}{c}\text { n } \\
\text { (árvores) }\end{array}$ & $\begin{array}{c}\text { Amostras } \\
\text { (\%) }\end{array}$ \\
\hline $\begin{array}{c}\text { Fazenda Pau- } \\
\text { Rainha }\end{array}$ & 1 & 74,7 & 116 & 46 & 39,7 \\
$\begin{array}{c}\text { Fazenda Pau- } \\
\text { Rainha }\end{array}$ & 2 & 90,7 & 123 & 46 & 37,4 \\
$\begin{array}{c}\text { Fazenda } \\
\text { Mundo Novo } \\
\text { Fazenda } \\
\text { Mundo Novo }\end{array}$ & 3 & 116,3 & 54 & 37 & 68,5 \\
\hline TOTAL & 108,9 & 59 & 38 & 64,4 \\
\hline
\end{tabular}

$\mathrm{DAP}=$ diâmetro a $1,30 \mathrm{~cm}$ do solo; $\mathrm{N}=$ número total de castanheiras na parcela de 9 ha; $n=$ número de castanheiras amostradas (árvores consideradas produtivas) na parcela de 9 ha.

As varáveis utilizadas para descrever o solo foram obtidas em apenas uma ocasião (em 2010) com a obtenção de amostras de solo até a profundidade de 80 cm na área de projeção das copas das árvores amostras. As variáveis de solos analisadas foram a profundidade, o alagamento e a textura.

Solos sem a presença de concreções (cascalho) até 80 $\mathrm{cm}$ de profundidade foram classificados como profundos e os demais pouco profundos. O alagamento foi avaliado observando-se a proximidade da árvore amostrada aos igarapés ou rios intermitentes, sua posição no relevo descolorações e manchas cinzentas no solo sondado, podendo ser classificadas como: árvores crescendo em local sem alagamento; sujeitos a acumulação de água, mas com boa drenagem e sujeitos a alagamento ao menos em uma época do ano.

Com base na coloração e na textura determinada pelo tato no campo, foram identificadas três classes de solo: vermelho arenoso; amarelo intermediário e amarelo argiloso. Essa classificação foi utilizada para análise das amostras no laboratório de solos da Embrapa Roraima para a determinação do percentual de argila, silte e areia pelo método de Bouyoucos. A análise de laboratório permitiu definir três classes de solo: solo 1: textura franco argilosa arenosa; solo 2: textura argilo-arenosa; solo 3: textura argilosa.

Os dados de precipitação foram obtidos em estação climatológica do Instituto Nacional de Meteorologia (INMET), instalada na cidade de Caracarai $\left(01^{\circ} 49^{\prime} 48,0^{\prime}\right.$ 'N e $61^{\circ} 07^{\prime} 12,0$ '” W).

Utilizando estes dados, um período de precipitação (mês ou conjunto de meses) foi selecionado com base na maior correlação (correlação de Pearson) com a produção em peso de sementes. Avaliamos a correlação entre o peso de sementes em 136 períodos distintos de precipitação, incluindo a precipitação mensal do mês de maio do ano anterior à queda (dispersão) dos frutos até o mês de julho do ano em que a dispersão ocorreu, bem como diversas combinações entre estes meses agrupados dois a dois, três a três e assim por diante, até a precipitação total acumulada entre maio e julho do ano seguinte. Incluímos também a precipitação mínima e máxima do período.

Para a modelagem utilizamos a análise de variância univariada no procedimento GLM (General Linear Model Univariate). Como variável dependente, incluímos a variável peso fresco de sementes ( $\mathrm{kg}$ árvore ${ }^{-1}$ ano $^{-1}$ ), e como independentes as variáveis qualitativas (alagamento, profundidade e tipo de solo) e como fator fixo a variável quantitativa (precipitação) como covariável.

No primeiro passo iniciamos com o modelo completo com as interações e a cada passo foram eliminadas as variáveis com valor de $F$ não significativo $(p \geq 0,05)$. Este procedimento foi repetido até a obtenção de um modelo totalmente significativo e, na sequência, observados se os parâmetros estimados para o modelo foram significativos pelo teste T. Quando isto não ocorreu, realizamos um novo agrupamento para esta variável.

\section{Resultados}

Foram observados cinco períodos de precipitação com maiores valores de correlação com a produção de sementes (Tabela 2). A maior correlação foi observada entre o peso de sementes ( $\mathrm{kg}$ arvore ${ }^{-1}$ ano $\left.^{-1}\right)$ e as precipitações no mês de setembro, que se caracteriza por uma sensível diminuição na precipitação no estado de Roraima. Desta forma, observamos que maiores precipitações neste mês estão associadas às maiores produções de sementes. 
Tabela 2. Coeficiente de correlação linear de Pearson para o peso fresco de sementes de Bertholletia excelsa em diferentes períodos de precipitação entre 2006 a 2010, em Caracaraí, RR.

Table 2. Pearson's linear correlation coefficient for fresh seed weight of Bertholletia excelsa in different periods of precipitation between 2006 and 2010, in Caracaraí, Roraima State.

\begin{tabular}{cccccc}
\hline & $\mathbf{P P}_{\text {set }}$ & $\mathbf{P P}_{\text {set-abr }}$ & $\mathbf{P P}_{\text {out-abr }}$ & $\mathbf{P P}_{\text {nov-abr }}$ & $\mathbf{P P}_{\text {feb }}$ \\
\hline Peso & 0,2288 & 0,2269 & 0,2240 & 0,2173 & $-0,2168$ \\
Sig. & 0,0000 & 0,0000 & 0,0000 & 0,0000 & 0,0000 \\
\hline
\end{tabular}

Peso: peso fresco de sementes (kg árvore ${ }^{-1}$ ano $^{-1}$ ); $\mathrm{PP}_{\text {set }}$ : precipitação total no mês de setembro; $\mathrm{PP}_{\text {set-abr }}$ : precipitação total entre os meses de setembro a abril; $\mathrm{PP}_{\text {out-ab: }}$ : precipitação total entre os meses de outubro a abril; $\mathrm{PP}_{\text {nov-abr }}$ : precipitação total entre os meses de novembro a abril; $\mathrm{PP}_{\mathrm{feb}}$ : precipitação total no mês de fevereiro.

A modelagem do peso fresco de sementes em função das variáveis de solo e precipitação no mês de setembro avançou sete passos e eliminou as variáveis, conforme apresentado na Tabela 3.

Após sete passos, foram mantidas as variáveis tipo de solo e interações profundidade $\mathrm{x}$ precipitação e tipo de solo x precipitação. A variável tipo de solo foi excluída, uma vez que não apresentou diferenças significativas para os parâmetros estimados. Na sequência, os dados dos solos tipo 1 e 2 foram unidos (solos tipo 1) e procedeu-se nova análise.
Tabela 3. Valor e significância de F para as variáveis de solo excluídas do modelo de produção de peso fresco de sementes de Bertholletia excelsa $\left(\mathrm{kg}_{\text {arvore }}{ }^{-1}\right)$ nos passos da modelagem.

Table 3. Value and significance of $F$ for the soil variables excluded from the model of production of fresh seed weight of Bertholletia excelsa $\left(\mathrm{kg}\right.$ tree $\left.^{-1}\right)$ in the modeling steps.

\begin{tabular}{clcc}
\hline Passo & \multicolumn{1}{c}{ Variável excluida } & F & Sig. \\
\hline 1 & Tipo de solo x profundidade & 0,098 & 0,755 \\
2 & Profundidade & 1,275 & 0,259 \\
3 & Tipo de solo x alagamento & 2,258 & 0,134 \\
4 & Alagamento & 1,293 & 0,256 \\
5 & Alagamento x precipitação & 2,258 & 0,134 \\
6 & $\beta_{0}$ (intercepto) & 1,771 & 0,184 \\
7 & Alagamento x profundidade & 2,466 & 0,062 \\
\hline
\end{tabular}

Os valores ajustados para o modelo de predição do peso fresco de sementes em função das propriedades do solo e precipitação estão apresentados na Figura 1. Podese constatar que a interação entre o tipo de solo com a precipitação teve mais influência sobre o peso fresco de sementes do que a interação profundidade do solo e precipitação. Observou-se ainda que a precipitação teve menor influência sobre o peso fresco em solos rasos.

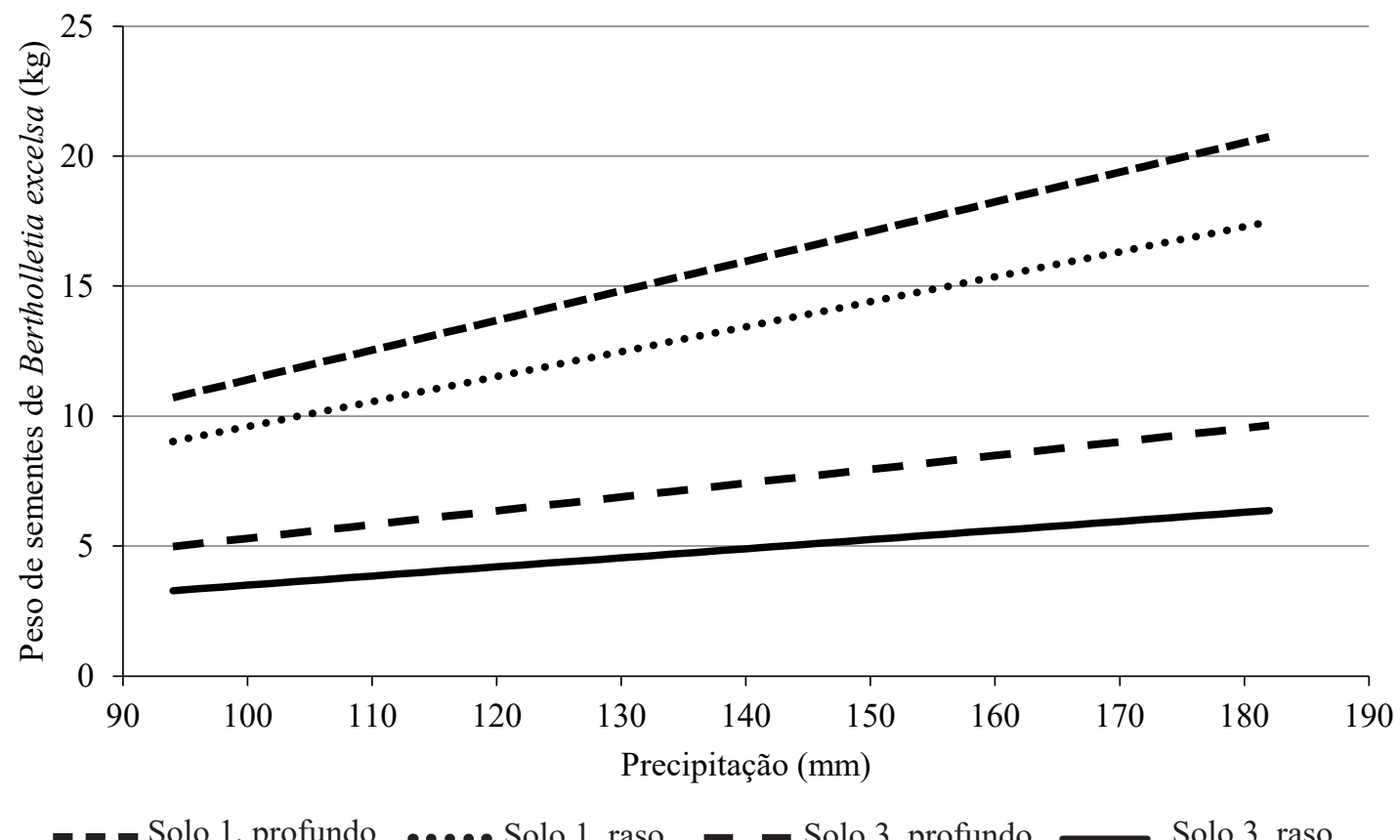

- - - Solo 1, profundo ...... Solo 1, raso - - Solo 3, profundo — Solo 3, raso

Figura 1. Predição para o peso fresco de sementes de Bertholletia excelsa em relação às propriedades do solo e precipitação.

Figure 1. Prediction of fresh weight of Bertholletia excelsa in relation to soil properties and precipitation. 


\section{Discussão}

As grandes variações de produção observadas em castanhais nativos na Amazônia dificultam a modelagem com o objetivo de prognosticar a produção de frutos e sementes em florestas nativas. Autores como Tonini \& Pedroso (2014) afirmaram que os fatores globais como as variações climáticas teriam grande efeito sobre o padrão de produção nas populações, porém a magnitude destas variações, mesmo em populações relativamente próximas, seriam controladas por fatores locais, como solo, relevo, densidade e estrutura da floresta, abundância de polinizadores, entre outros.

Dos fatores de produção locais, os teores de nutrientes no solo parecem ter pouca influência sobre a variação na produção de frutos da castanheira-do-brasil em florestas nativas (Kainer et al., 2007; Silva et al., 2015; Costa et al., 2017), podendo seu efeito ser encoberto por variáveis como as dimensões do tronco (DAP), atributos da copa, e a precipitação e suas interações com o tipo e a profundidade do solo que, neste estudo (Figura 1), se mostraram importantes para a predição da produção de sementes de castanheira-do-brasil e devem ser consideradas em estudos de mesma natureza.

Este resultado corrobora com Kainer et al. (2007) que afirmaram que o clima é o principal responsável pelas variações de produção de frutos em espécies da floresta tropical, levantando a hipótese de que estas variações devem seguir os padrões de precipitação ou de temperatura. Os autores também encontraram correlação significativa entre o número de frutos produzidos e a precipitação acumulada nos cinco meses de estação seca (maio a setembro). No entanto, a correlação foi mais fraca na comparação com nosso estudo, provavelmente devido à falta de dados mais precisos, em função da distância da estação meteorológica em relação a área do estudo (no caso $200 \mathrm{~km}$ ).

Os frutos da castanheira-do-brasil necessitam de longo tempo para o seu desenvolvimento, em alguns casos mais do que 12 meses (Maués, 2002; Zuidema, 2003; Tonini, 2011) e, desta forma, quando se considera a precipitação como variável explicativa para as variações de produção, a questão passa a ser qual o período deve ser considerado.

Especula-se que a precipitação no período de emissão dos botões florais afeta a produção de sementes de castanha, uma vez que as correlações mais importantes entre estas variáveis (Tabela 2 ) referem-se normalmente aos meses iniciais da emissão de botões florais, que ocorre entre outubro e dezembro, com máxima emissão de botões florais em fevereiro para o estado de Roraima (Tonini, 2011).

É necessária maior investigação sobre o papel da precipitação na produção de sementes, bem com a inclusão de outras variáveis ambientais, como a temperatura, para melhor compreensão da produção de sementes. Sunil et al. (2011) reportam um modelo para a predição do peso fresco de nozes de Areca catechu, empregando como variáveis independentes a precipitação e a temperatura. Rathcke \& Lacey (1985) afirmaram que fatores abióticos, como a precipitação, podem interferir sobre a floração de espécies florestais, por afetarem a habilidade de uma planta em produzir flores ou por afetarem, indiretamente, os polinizadores. E autores como Zuidema (2003) e Kainer et al. (2007) comentaram sobre redução na produção de frutos de árvores de castanheira-do-brasil com a redução da precipitação em anos de seca prolongada, ocasionadas pelo "El Niño".

Os resultados obtidos apontam que árvores de castanheira-do-brasil crescendo em solos franco argiloarenosos e argilo-arenosos profundos apresentam maior produção de sementes de castanha, indo de encontro ao reportado por Locatelli et al. (2003) como habitat preferencial da espécie os solos profundos, bem estruturados e drenados, quase sempre argilosos ou argilo-arenosos.

As árvores de castanheira-do-brasil crescendo em solos argilo-arenosos profundos e franco-argilosos e argilo-arenosos rasos apresentaram produção média de sementes de castanha, e as que cresciam em solos argilosos rasos apresentaram as menores produções de sementes, corroborando com Lima et al. (2015), que ao estudarem a relação entre as variáveis físicas e químicas do solo e o crescimento da espécie em plantios, observaram melhor desempenho em solos argilosos. Solos com altos teores de silte e areia tiveram influência negativa sobre o crescimento da espécie.

\section{Conclusões}

Maiores precipitações em setembro, caracterizado por ser o mês de transição entre a estação seca e a chuvosa, se correlacionaram de forma significativa e positiva com a produção de sementes.

Árvores de castanheira-do-brasil crescendo em solos rasos de textura argilosa produzem menos sementes, 
quando comparadas a árvores crescendo em solos profundos de textura franco-argilosa e argilo-arenosa.

\section{Agradecimentos}

Os autores agradecem ao Conselho Nacional de Desenvolvimento Científico e Tecnológico (CNPq)processo 575393/2008-4 pelo apoio financeiro.

\section{Referências}

Coollinson, C. et al. Economic viability of Brazil Nut trading in Peru. United Kingdon: University of Greenwich, 2000. 64 p.

Costa, M. C. et al. Atributos do solo relacionados com a produção de castanheira. Floresta e Ambiente, v. 24, e20150042, 2017. DOI: 10.1590/2179-8087.004215.

Fundação do Meio Ambiente Ciência e Tecnologia do Estado de Roraima. O Brasil do Hemisfério Norte: diagnóstico científico e tecnológico para o desenvolvimento. Boa Vista, 1993. 512 p.

Homma, A. K. O. et al. Castanheira-do-pará: os desafios do extrativismo para plantios agrícolas. Boletim do Museu Paraense Emilio Goeldi, v. 9, n. 2, p. 293-306, 2014.

IBGE. Produção da extração vegetal e da silvicultura. Rio de Janeiro, 2016. v. 31.

Instituto de Manejo e Certificação Florestal e Agrícola. Panorama nacional da cadeia de valor da castanha-do-brasil. Piracicaba, SP, 2016. $60 \mathrm{p}$.

Kainer, K. A. et al. Explaining variation in Brazil nut fruit production. Journal of Tropical Ecology, v. 22, p. 147-154, 2007. DOI: 10.1016/j.foreco.2007.05.024.

Lima, R. M. B. Influência dos fatores edáficos no crescimento de Bertholletia excelsa H.B.K na Amazônia. In: CONGRESSO BRASILEIRO DE SISTEMAS AGROFLORESTAIS, 5, 2005. Curitiba. Anais.... Colombo: Embrapa Florestas, 2005. p. 319-321.

Locatelli, M. et al. Castanha-do-Brasil: opção para solo de baixa fertilidade na Amazônia. In: SEMINÁRIO NACIONAL DEGRADAÇÃO E RECUPERAÇÃO AMBIENTAL, 2003, Foz do Iguaçu. Anais... Foz do Iguaçu: Sobrade, 2003. p. 1-7.
Lorenzi, H. Árvores brasileiras. 4. ed. São Paulo: Instituto Plantarum, 2000. v. 1.384 p.

Maués, M. M. Reproductive phenology and pollination of the Brazil nut tree (Bertholletia excelsa Humb. \& Bonpl. lecythidaceae) in Eastern Amazonia. In: WORKSHOP ON THE CONSERVATION AND SUSTAINABLE USE OF POLLINATORS IN AGRICULTURE, WITH EMPHASIS ON BEE, 1998, São Paulo. Pollinating bees: the conservation link between agriculture and nature: proceedings. Brasília: Ministry of Environment, 2002. p. 245-254.

Müller, C. H. \& Caizavara, B. B. G. Castanha-do-brasil. Belém: CPATU, 1989. 6 p. (Informações básicas, 11).

Ortiz, E. G. Brazil nut. In: Shanley, P.; Pierce, A. R.; Laird, S. A.; Guillen, A. (Ed.). Tapping the green market: certification and management of non-timber forest products. London: Earthscan, 2002. p. 61-74. (Peaple and plants conservation series).

Rathcke, B. \& Lacey, E. P. Phenological patterns of terrestrial plants. Annual Review of Ecology and Systematics, v. 16, p. 179-214, 1985. DOI: 0066-4 162/85/1120-0179\$02.00.

Silva, M. F. et al. Correlação entre teores de nutrientes do solo, foliar e produção da castanha-do-brasil na Amazônia sul ocidental. In: REUNIÃO DE CIÊNCIA DO SOLO DA AMAZÔNIA OCIDENTAL, 2., 2014, Porto Velho. Anais... Porto Velho: Núcleo Regional Amazônia Ocidental da Sociedade Brasileira de Ciência do Solo, 2015. p. 183-186.

Sunil, K. M. et al. Influence of weather parameters on yield and yield attributes of areca nut (Areca catechu L.). Journal of Agricultural Physics, v. 11, p. 88-90, 2011.

Tonini, H. Fenologia da castanheira-do-brasil (Bertholletia excelsa Humb. \& Bonpl., Lecythidaceae) no sul do estado de Roraima. Cerne, v. 17, n. 1, 2011. DOI: 10.1590/S0104-77602011000100015.

Tonini, H. \& Pedroso, C. A. Variações anuais na produção de frutos e sementes de Castanheira-do-Brasil (Bertholletia excelsa Bonpl., Lecythidaceae) em florestas nativas de Roraima. Revista Árvore, v. 38, n. 1, p. 133-144, 2014. DOI: 10.1590/S010067622014000100013.

Zuidema, P. A. Ecology and management of the brazil nut tree (Bertholletia excelsa). Utecht: Promab, 2003. 111 p. (Scientific series, 6). 